\title{
Environmental Social Sciences: Methods and Research Design
}

\author{
Ismael Vaccaro, Eric Alden Smith, and Shankar Aswani, eds. 2010. Cambridge University Press, Cambridge. Pp. \\ 396, 41 b/w illustrations, 20 tables. US\$49.99 (paperback). ISBN 9780521125710.
}

Reviewed by Jeremy Spoon

Reviewer Address: 724 Southwest Harrison Street, Portland, Oregon, 97201

Received: March 11, 2012

Volume 3:33-34

Published: June 26, 2012

(C) 2012 Society of Ethnobiology

Research on human-environment relationships necessitates an interdisciplinary lens and mixed methodologies. Environmental Social Sciences: Methods for Research Design, edited by Vaccaro, Smith, and Aswani, provides a framework and toolkit for engaging complex social and ecological questions. This book will assist researchers interested in the social components of environmental issues to identify social science research methods through contemporary examples, both quantitative and qualitative. It accessibly links theory to method and utilizes relevant case studies to illustrate the proposed techniques in a variety of contexts. The volume also clearly identifies how these methods correspond and some of the challenges with these approaches.

Researchers often piece together social science and other methods to answer complex, interrelated social, cultural, and environmental questions in diverse spatial and temporal contexts. The methods described in this book unite techniques and express linkages for operationalization across the social sciences, humanities, and natural sciences. Ethnobiologists will find this work useful for a myriad of research focuses, from cognitive studies on local ecological knowledge and perceptions of landscape to multi-scale analyses of market influences on resource use and management.

Vaccaro and Smith argue that environmental social science requires a consideration of past and present human communities and their relationships with the physical environments that support(ed) them. Research emphases and theoretical orientations include economic factors embedded in the study of cultural ecology, analysis of the social construction of knowledge in ethnobiology and science studies, politics and ideology in political ecology, and institutions in property and collective action theories, among others. The social sciences and humanities thus offer complementary methods to understand the social aspects of human-environment relationships across spatial and temporal scales. These techniques are both quantitative and qualitative, and combine discursive, statistical, and spatial analyses.

No single methodological approach or causality offers an understanding of an environmental issue. The very fact that environment problems can be assessed from multiple perspectives does not assure the integration of research. The starkest divide has opposed "scientific or positivist approaches to critical, subjectivist, or postmodern schools" (4). This volume challenges this dichotomy and offers a framework that links quantitative and qualitative methods to examine the complexity of environmental issues in new and creative ways.

The book highlights 14 parallel themes related to environmental social science research and offers an integrated applied example as the final chapter. The themes include: demography, household production decisions, property theory and institutional analyses, political economy, ethnobiology, mapping histories, literary analyses, text analysis, social networks, multisited ethnography, geospatial analysis, archaeological methods, historical ecology, and conservation design. While a single chapter focuses specifically on ethnobiology methods in practice from voucher collection and photo elicitation to domain analysis and participatory mapping using cases studies from Tibet, North America, and Brazil (Chapter 6), multiple chapters are relevant to ethnobiological inquiry. Select examples follow.

Beltran (Chapter 2) illustrates how demography assists in understanding a social and environmental context. Demography in general provides a "road map" of a population, which can be correlated with ethnographic data, such as ethnobiological knowledge or perceptions of landscape change. Demographic 
indicators become "touchstones" that can enhance the elucidation of political and economic drivers of environmental relationships and the changing factors that shape landscapes. Demographic and ethnographic data can thus be layered to form a better understanding of causation. For example, demographic variables can be used to assess ecological knowledge distribution and change.

Zonotti, Glover, and Sepez (Chapter 6) describe a variety of mixed ethnobiological methods used to examine contemporary ethnobiology and associated place based knowledge. Ethnobiologists will find this chapter especially useful for articulating a host of methods that can be used for a variety of research questions. These methods include: the collection of voucher specimens, flora and fauna identification and classification, cultural consensus analysis, ethnosemantics, free listing, pile sorts and triads, various interview techniques, photo elicitation and interpretation, and participatory mapping and plant trails. These techniques cover an entire spectrum of ethnographic inquiry - structured and unstructured, in situ and ex situ, quantitative and qualitative.

Strang (Chapter 7) expresses cultural mapping as an ethnographic method that explores historical and contemporary relationships with local environments. This approach takes participants on "walkabouts" in places considered locally important and collects social, historical, and ecological data in situ. In this method, places are considered as repositories of information and as mnemonic devices, drawing upon both experiential and abstract knowledge. Ethnobiologists interested in place based knowledge of species, habitats, or watersheds, ownership and rights of access, economic practices and resource use and management can benefit from this approach.
Johnson and Griffith (Chapter 10) discuss research exploring the relationships between systems of human relations (or social networks) and ecosystem interactions. Social networks assist in understanding the sharing and distribution of ecological and environmental knowledge, resource allocation, and adaptive responses to stochastic events and climate change. Social network analysis offers ethnobiologists a lens to understand the heterogeneity of a human population and how and why knowledge and practice change through research on the social connections among social entities. Social network analysis is especially promising for ethnobiological research on the transmission and dynamics of cognitive information.

The book lacks a discrete ethics section and has a scant discussion on theory in the introduction. My perspective is that all social science methods texts should contain a discussion of ethics, especially related to intellectual property and applied research. Many chapters utilize theory to articulate methods; however a reader would also benefit from a more comprehensive review of theory in the introduction or in a separate chapter.

Overall, this book is a welcome contribution to the interdisciplinary study of human-environment relationships. It succinctly and accessibly introduces several quantitative and qualitative methods from the social sciences and skillfully animates the methodologies with well-chosen case studies. The 14 themes are useful entry points for students, scholars, and practitioners to construct a methodological toolkit for context specific questions at multiple scales. As research on environmental issues continues to increase, future studies will indeed benefit from these interdisciplinary approaches and mixed methods. 